\title{
MicroRNA-889-3p targets FGFR2 to inhibit cervical cancer cell viability and invasion
}

\author{
YU SUN, YAN CHENG, YAN ZHANG and KUN HAN \\ Department of Obstetrics and Gynecology, Weifang People's Hospital, Weifang, Shandong 261041, P.R. China
}

Received September 8, 2018; Accepted May 2, 2019

DOI: $10.3892 / \mathrm{etm} .2019 .7675$

\begin{abstract}
MicroRNAs (miRNAs) are frequently dysregulated in cervical cancer, and the aberrant regulation of miRNAs may be involved in the regulation of various cancer-associated biological processes. Therefore, further exploration of the specific roles of dysregulated miRNAs in cervical cancer and their associated mechanism may promote the development of effective therapeutic approaches. miRNA-889-3p (miR-889) serves crucial roles in esophageal squamous cell carcinoma and hepatocellular carcinoma. However, to the best of our knowledge, no studies concerning the relationship between miR-889 and cervical cancer were performed. The aims of this study were to measure miR-889 expression in cervical cancer and to examine the potential effects of miR-889 in cervical cancer development on a molecular level to provide potential clinical insight. The present study revealed that miR-889 was downregulated in cervical cancer tissues and cell lines. Reduced miR-889 expression was significantly associated with International Federation of Gynecology and Obstetrics cancer staging and with lymph node metastasis. In addition, miR-889 overexpression reduced cervical cancer cell viability and invasive ability. Using bioinformatics analysis, fibroblast growth factor receptor 2 (FGFR2) was predicted to be a potential target of miR-889, which was confirmed using luciferase reporter assay. Reverse transcription-quantitative PCR and western blot analysis results suggested that miR-889 overexpression decreased FGFR2 expression in cervical cancer cells at the mRNA and the protein level, respectively. Conversely, FGFR2 silencing using small interfering RNA imitated the tumor suppressive effects of miR- 889 overexpression in cervical cancer cells, which was successfully reversed by plasmid-facilitated FGFR2 overexpression. These observations demonstrated that miR-889 may serve tumor suppressive roles in cervical cancer by directly targeting FGFR2, which
\end{abstract}

Correspondence to: Professor Kun Han, Department of Obstetrics and Gynecology, Weifang People's Hospital, 151 Guangwen Road, Weifang, Shandong 261041, P.R. China

E-mail: kunhan_wf@yeah.net

Key words: cervical cancer, microRNA-889-3p, fibroblast growth factor 2, viability, invasion indicated that this miRNA may be a promising therapeutic target for patients with cervical cancer.

\section{Introduction}

Cervical cancer is one of the most common gynecological malignancies and is currently the fourth most common cause of cancer-associated mortality worldwide (1). According to data obtained from Global cancer statistics (2), it is estimated that there were 569,847 new cases and 311,365 mortalities in 2018 as a result of cervical cancer.

Although human papillomavirus (HPV) infection has been demonstrated to be closely associated with the pathogenesis of cervical cancer, HPV alone cannot be accounted for as the sole cause of this disease $(3,4)$. Clinical outcomes for cervical cancer remain unsatisfactory despite improvements in diagnostic techniques and therapeutic approaches, including surgery, radiotherapy and chemotherapy (5). The primary reasons for poor prognosis in patients with cervical cancer include recurrence, invasion and metastasis (6). Therefore, it is of urgent concern to fully elucidate the mechanism underlying the aggressive pathophysiology of this disease, to identify effective therapeutic interventions and to improve patient prognosis.

In the past few decades, microRNAs (miRNAs) have been implicated in tumorigenesis and tumor development (7-9). miRNAs are a class of non-coding short RNA molecules, 18-24 nucleotides in length (10) that regulate gene expression by binding to target sites in the 3'-untranslated regions (3'-UTRs) of target mRNAs, resulting in translation suppression and/or mRNA degradation (11). To date, $>2,500$ miRNAs have been identified in the human genome (12), with $\sim 60 \%$ of protein-coding genes reported to be targeted by miRNAs (13). Dysregulation of miRNA function has been documented in nearly all types of human malignant tumors such as cervical cancer (14-16). miRNAs may be involved in the modulation of cervical carcinogenesis and progression by acting as either oncogenes or tumor suppressors (17-19). Therefore, unravelling the downstream consequences of miRNA dysregulation in cervical cancer is of clinical importance for the identification of effective biomarkers for the diagnosis and prognosis of cervical cancer in addition to the development of novel therapeutic approaches for patients with this malignancy.

microRNA-889-3p (miR-889) has been previously demonstrated to serve crucial roles in esophageal squamous cell 
carcinoma (20) and hepatocellular carcinoma (21). However, to the best of our knowledge, no studies concerning the relationship between miR-889 and cervical cancer have been performed. Therefore, the aim of this study was to investigate miR-889 expression in cervical cancer and its clinical significance, and to examine the potential effects of miR-889 in cervical cancer development on a molecular level. Expression and functions of fibroblast growth factor receptor 2 (FGFR2) have been extensively studied in multiple human cancer types (22-25). FGFR2 is highly expressed in cervical cancer tissues (26). In the present study, FGFR2 was predicted as a potential target of miR-889. A series of experiments were performed to validate whether FGFR2 was a direct target gene of miR-889 in cervical cancer, and whether a decrease of FGFR2 was responsible for the activity of miR-889 overexpression in cervical cancer cells.

\section{Materials and methods}

Ethical approval and collection clinical tissues. This study was approved by the Ethics Committee of Weifang People's Hospital (Weifang, China), and written informed consent was provided by all individuals who participated in the present study. In total, 49 pairs of cervical cancer tissues and matched non-cancerous tissues were obtained from patients with cervical cancer (age range, 46-71 years) following surgical resection at Weifang People's Hospital between June 2015 and August 2017. The inclusion criteria were patients diagnosed with cervical cancer and treated with surgical resection. In addition, none of the patients received radiotherapy or chemotherapy prior to surgery. Patients treated with preoperative radiotherapy or chemotherapy were excluded from this study. All tissues were promptly snap-frozen in liquid nitrogen following surgical resection and stored at $-80^{\circ} \mathrm{C}$ until further use.

Cell lines and culture conditions. A total of four human cervical cancer cell lines (HeLa, C-33A, CaSki and SiHa) and a normal human cervical epithelial cell line (Ect1/E6E7) were purchased from American Type Culture Collection. All cell lines were maintained in Dulbecco's modified Eagle's medium (DMEM) supplemented with 10\% FBS, $100 \mathrm{U} / \mathrm{ml}$ penicillin and $100 \mu \mathrm{g} / \mathrm{ml}$ streptomycin (all from Invitrogen; Thermo Fisher Scientific, Inc.) in a humidified atmosphere at $37^{\circ} \mathrm{C}$ under $5 \% \mathrm{CO}_{2}$.

Cell transfection. The miR-889 mimic and negative control miRNA mimic (miR-NC) were chemically constructed by Guangzhou RiboBio Co., Ltd. The miR-889 mimics sequence was 5'-UUAAUAUCGGACAACCAUUGU-3' and the miR-NC sequence was 5'-UUCUCCGAACGUGUCACGUTT-3'. Small interfering RNA (siRNA) targeting FGFR2 and negative control siRNA (NC siRNA) were purchased from Shanghai GenePharma Co., Ltd. The FGFR2 siRNA sequence was 5'-GGAGGUGCUUCACUUAAGATT-3' and the NC siRNA sequence was 5'-UUCUCCGAACGUGUCACGUTT-3'. The pcDNA3.1-FGFR2 overexpression vector (pc-FGFR2) lacking its 3'-UTR, and the empty pcDNA3.1 vector were purchased from the Chinese Academy of Sciences. Cells were seeded $\left(6 \times 10^{5}\right.$ cells/well) into 6 -well plates $24 \mathrm{~h}$ prior to transfection and subsequently transfected with miR-889 mimic (100 pmol), miR-NC (100 pmol), FGFR2 siRNA (100 pmol), NC siRNA (100 pmol), pc-FGFR2 $(4 \mu \mathrm{g})$ or empty pcDNA3.1 vector $(4 \mu \mathrm{g})$ using Lipofectamine ${ }^{\circledR} 2000$ reagent (Invitrogen; Thermo Fisher Scientific, Inc.). Transfection was performed at room temperature according to manufacturer's protocols. Reverse transcription-quantitative PCR (RT-qPCR) and western blot analysis were conducted 48 and $72 \mathrm{~h}$ following transfection, respectively. Cell Counting Kit-8 (CCK-8) assay was performed on transfected cells $24 \mathrm{~h}$ after transfection, whereas invasion assay was conducted $48 \mathrm{~h}$ following transfection.

$R T-q P C R$. For the measurement of miR-889 and FGFR2 mRNA expression, total RNA was isolated from tissues $(50 \mathrm{mg}$ ) or cells $\left(1 \times 10^{6}\right)$ using TRIzol ${ }^{\circledR}$ reagent (Invitrogen; Thermo Fisher Scientific, Inc.) according to manufacturer's protocol. A Nanodrop 2000 spectrophotometer (Thermo Fisher Scientific, Inc.) was utilized to assess the concentration and quality of total RNA. For the detection of miR-889 expression, total RNA was reverse-transcribed into cDNA using miScriptII Reverse Transcription Kit (Qiagen China Co., Ltd.) according to the manufacturer's protocol. The cDNA was subsequently subjected to qPCR using miScript SYBR ${ }^{\circledR}$ Green PCR Kit (Qiagen China Co., Ltd.) according to manufacturer's protocol. The thermocycling conditions for qPCR were as follows: $95^{\circ} \mathrm{C}$ for $2 \mathrm{~min}, 95^{\circ} \mathrm{C}$ for $10 \mathrm{sec}, 55^{\circ} \mathrm{C}$ for $30 \mathrm{sec}$ and $72^{\circ} \mathrm{C}$ for $30 \mathrm{sec}$, for 40 cycles.

For the measurement of FGFR 2 mRNA expression, cDNA was generated from RNA using Prime-Script Reverse Transcription Reagent Kit (Takara Biotechnology Co., Ltd.) according to the manufacturer's protocol. qPCR was performed using SYBR Premix Ex Taq ${ }^{\mathrm{TM}}$ Kit (Takara Biotechnology Co., Ltd.) according to the manufacturer's protocol. The thermocycling conditions for qPCR were as follows: $5 \mathrm{~min}$ at $95^{\circ} \mathrm{C}$, followed by 40 cycles of $95^{\circ} \mathrm{C}$ for $30 \mathrm{sec}$ and $65^{\circ} \mathrm{C}$ for $45 \mathrm{sec}$. U6 small nuclear RNA and GAPDH served as loading controls and for normalization of miR-889 and FGFR2 mRNA expression, respectively. The $2^{-\Delta \Delta \mathrm{Cq}}$ method was applied to quantify relative gene expression (27). The following primer pairs were used for the qPCR: miR-889, forward 5'-ACACTCCAG CTGGGTTAATATCGGACAAC-3' and reverse 5'-TGGTGT CGTGGAGTCG-3'; U6, forward 5'-CTCGCTTCGGCAGCA CA-3' and reverse 5'-AACGCTTCACGAATTTGCGT-3'; FGFR2, forward 5'-CGCTGGTGAGGATAACAACACG-3' and reverse 5'-TGGAAGTTCATACTCGGAGACCC-3'; and GAPDH, forward 5'-AACGGATTTGGTCG-TATTG-3' and reverse 5'-GGAAGATGGTGATGGGATT-3'.

CCK- 8 assay. A total of $100 \mu 1$ culture medium containing $\sim 3,000$ cells were seeded into each well of 96-well plates $24 \mathrm{~h}$ after transfection. Five replicates were set for every group. The culture plates were incubated at $37^{\circ} \mathrm{C}$ under $5 \%$ $\mathrm{CO}_{2}$ for $0,24,48$ and $72 \mathrm{~h}$ before $10 \mu \mathrm{l}$ of CCK- 8 solution (Dojindo Molecular Technologies, Inc.) was added into each well. Following a further $2 \mathrm{~h}$ of incubation, absorbance was measured at $450 \mathrm{~nm}$ using a microplate reader (Bio-Rad Laboratories, Inc.) to determine cell viability.

Invasion assay. The invasive ability of the cells was examined using 24-well Transwell ${ }^{\circledR}$ chambers (pore size, $8 \mu \mathrm{m}$; Corning Inc.) pre-coated with Matrigel (BD Biosciences). Cells were 
serum-starved for $12 \mathrm{~h}$ then harvested and resuspended in FBS-free DMEM prior to being seeded into the upper chambers at a density of $5 \times 10^{4}$ cells/well $48 \mathrm{~h}$ following transfection. A total of $500 \mu$ l DMEM supplemented with $10 \%$ FBS was added into the lower chambers, which served as the chemoattractant. Following $24 \mathrm{~h}$ of incubation, cells remaining on the upper side of the chambers were mechanically removed using a cotton swab. Cells that successfully invaded were fixed with $4 \%$ paraformaldehyde at room temperature for $30 \mathrm{~min}$, stained with $0.1 \%$ crystal violet at room temperature for $30 \mathrm{~min}$ and washed with PBS. The average number of invaded cells was counted using a light microscope (magnification, x200; Olympus Corporation) from five randomly chosen microscopic fields of view for each membrane.

Bioinformatics prediction. TargetScan (Release 7.2; http://www.targetscan.org/vert_72/) and miRDB (www. mirdb.org) were used to search for potential miR-889 targets. Hsa-miR-889-3p was entered into the searching bar and a list of potential target genes were obtained.

Dual-luciferase reporter assay. The wild-type (WT) miR-889 target site in the 3'-UTR of FGFR2 or a mutant (MUT) 3'-UTR were amplified by Shanghai GenePharma Co., Ltd. and inserted into the pMIR-GLO ${ }^{\text {TM }}$ Luciferase vector (Promega Corporation) downstream of the firefly luciferase coding region. The generated luciferase reporter plasmids were defined as pMIR-FGFR2-3'-UTR WT and pMIR-FGFR2-3'-UTR MUT thereafter. Cells were seeded into 24 -well plates at a density of $1 \times 10^{5}$ cells/well one day prior to transfection. miR-889 mimic (20 pmol) or miR-NC (20 pmol) were co-transfected with pMIR-FGFR2-3'-UTR WT (0.2 $\mu \mathrm{g})$ or pMIR-FGFR2-3'-UTR MUT $(0.2 \mu \mathrm{g})$ using Lipofectamine 2000, in accordance with the manufacturer's protocol. Co-transfection was performed at room temperature then co-transfected cells were incubated at $37^{\circ} \mathrm{C}$ under $5 \% \mathrm{CO}_{2}$ for $48 \mathrm{~h}$. Using a Dual-Luciferase Reporter Assay System (Promega Corporation), luciferase activity was assessed $48 \mathrm{~h}$ after incubation according to the manufacturer's protocol. Firefly luciferase activity was normalized to Renilla luciferase activity.

Western blot analysis. Cells $\left(1 \times 10^{6}\right)$ and tissues $(100 \mathrm{mg})$ were washed with PBS prior to protein extraction using radioimmunoprecipitation assay buffer (Sigma-Aldrich; Merck KGaA). Protein concentration was measured using Bicinchoninic Acid protein assay kit (Sigma-Aldrich; Merck $\mathrm{KGaA})$. Equal amounts of protein $(30 \mu \mathrm{g})$ were separated by $10 \%$ SDS-PAGE, transferred to polyvinylidene difluoride membranes and blocked at room temperature for $2 \mathrm{~h}$ with $5 \%$ non-fat milk powder diluted in Tris-buffered saline containing $0.1 \%$ Tween-20 (TBST). Following incubation overnight at $4^{\circ} \mathrm{C}$ with primary antibodies, the membranes were washed with TBST and incubated at room temperature for $1 \mathrm{~h}$ with horseradish peroxidase-conjugated goat anti-mouse secondary antibody (1:5,000 dilution; cat. no. ab205719; Abcam). To visualize the protein bands ECL ${ }^{\mathrm{TM}}$ Western Blotting Detection Reagents (GE Healthcare) was used. The primary antibodies used in this study were as follows: FGFR2 (1:1,000 dilution; cat. no. ab58201; Abcam) and GAPDH (1:1,000 dilution; cat. no. ab9482; Abcam). Quantity One software version 4.62
Table I. Association between miR-889 expression and clinicopathological features of patients with cervical cancer.

\begin{tabular}{lccc}
\hline & \multicolumn{2}{c}{ miR-889 expression } & \\
\cline { 2 - 3 } Clinicopathological feature & Low & High & P-value \\
\hline Age (years) & & & 0.444 \\
$\quad<55$ & 11 & 8 & \\
$\quad \geq 55$ & 14 & 16 & \\
Tumor size (cm) & & & 0.588 \\
$\quad<4$ & 8 & 6 & \\
$\quad \geq 4$ & 17 & 18 & \\
Family history of cancer & & & 0.413 \\
$\quad$ Yes & 4 & 2 & \\
$\quad$ No & 21 & 22 & \\
FIGO stage & & & $\mathbf{0 . 0 3 0}$ \\
$\quad$ I-II & 10 & 17 & \\
$\quad$ III-IV & 15 & 7 & \\
Lymph node metastasis & & & $\mathbf{0 . 0 1 2}$ \\
$\quad$ No & 11 & 19 & \\
Yes & 14 & 5 & \\
\end{tabular}

miR, microRNA; FIGO, International Federation of Gynecology and Obstetrics.

(Bio-Rad Laboratories, Inc.) was employed for performing densitometric analysis to quantify protein expression.

Statistical analysis. All data were presented as the mean \pm standard deviation from at least three separate experiments. SPSS software version 16.0 (SPSS, Inc.) was used to perform all statistical analyses. $\chi^{2}$ test was applied to determine the association between miR-889 expression and clinicopathological factors in patients with cervical cancer. Student's t-test was used to analyze differences between two groups. Differences between multiple groups were compared using one-way analysis of variance followed by Tukey's post-hoc test. $\mathrm{P}<0.05$ was considered to indicate a statistically significant difference.

\section{Results}

miR-889 expression is reduced in cervical cancer tissues and cell lines. RT-qPCR analysis was performed to measure miR-889 expression levels in 49 pairs of cervical cancer tissues and matched non-cancerous tissues. A significantly lower level of miR-889 expression was observed in cervical cancer tissues compared with their corresponding matched noncancerous tissues ( $\mathrm{P}<0.05$; Fig. 1A). Subsequently, the association between miR-889 and a number of clinicopathologic factors were investigated in patients with cervical cancer. All patients were divided into either low or high miR-889 expression groups using the median value of miR-889 in cervical cancer tissues as the cutoff value. Low miR-889 expression was demonstratively associated with International Federation of Gynecology and Obstetrics (FIGO) stages $(\mathrm{P}=0.030)$ and increased lymph node metastasis $(\mathrm{P}=0.012$; Table $\mathrm{I})$. 

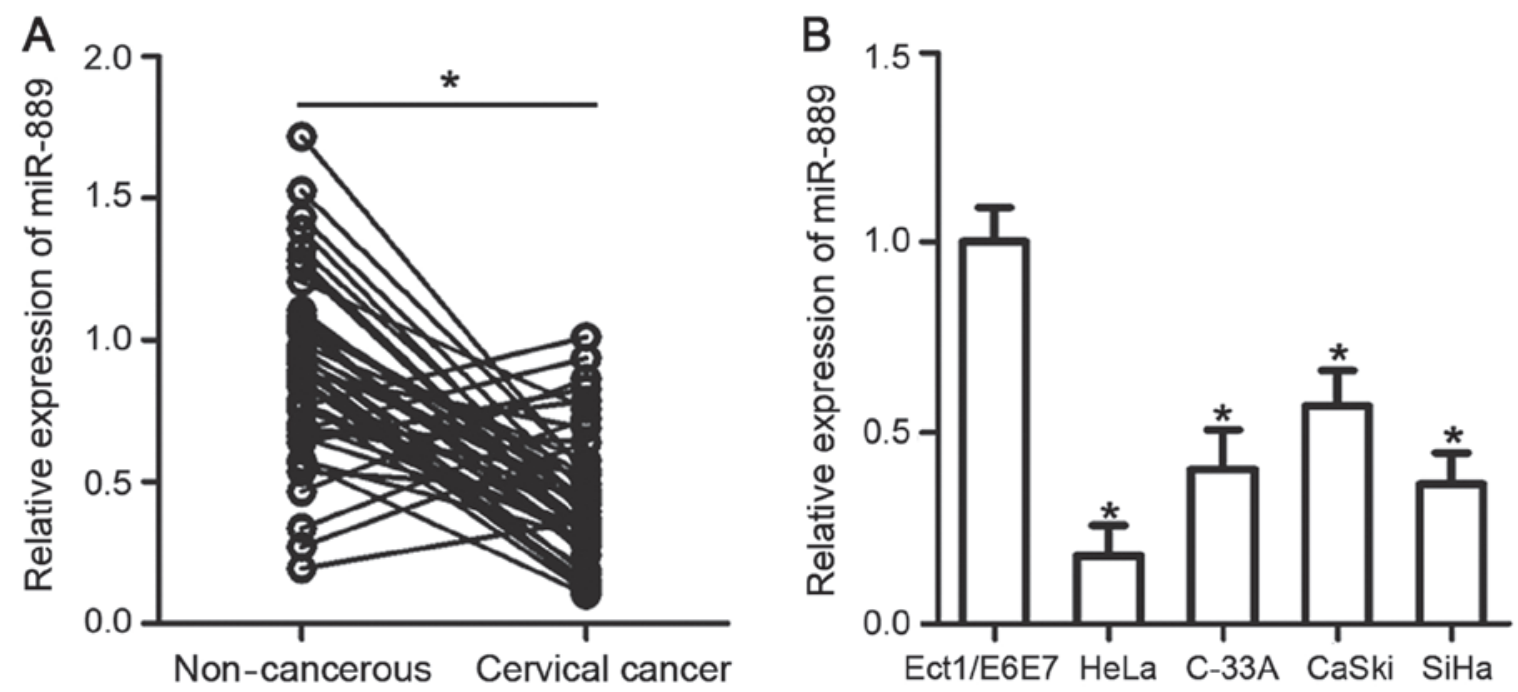

Figure 1. miR-889 expression levels are reduced in cervical cancer tissues and cell lines. (A) RT-qPCR was performed to measure and compare miR-889 expression levels in 49 pairs of cervical cancer tissues and matched noncancerous tissues. ${ }^{*} \mathrm{P}<0.05$ vs. non-cancerous. (B) Expression of miR-889 levels was determined in four human cervical cancer cell lines (HeLa, C-33A, CaSki and SiHa) and a normal human cervical epithelial cell line (Ect1/E6E7) by RT-qPCR. *P<0.05 vs. Ect1/E6E7. miR, microRNA; RT-qPCR, reverse transcription-quantitative polymerase chain reaction.

To support this observation, the level of miR-889 mRNA expression was measured and assessed in four human cervical cancer cell lines (HeLa, C-33A, CaSki and SiHa) and a normal human cervical epithelial cell line (Ect1/E6E7) using RT-qPCR. miR-889 was demonstrated to be significantly lower in all four of the cervical cancer cell lines compared with expression in Ect1/E6E7 cells ( $\mathrm{P}<0.05$; Fig. 1B). These results suggested that miR-889 expression is downregulated in cervical cancer, and may be linked with the development of this malignancy.

miR-889 inhibits cervical cancer cell viability and invasion. HeLa and SiHa cells exhibited the lowest expression levels of miR-889 among the four cervical cancer cell lines (Fig. 1B); therefore, these two cervical cancer cell lines were chosen for subsequent experiments. To assess the role of miR-889 in cervical cancer malignant phenotype, HeLa and SiHa cells were transfected with either the miR-889 mimic or miR-NC. Successful upregulation of miR-889 expression after miR-889 mimic transfection was verified in the two cell lines using RT-qPCR $(\mathrm{P}<0.05$; Fig. 2A). The effect of miR-889 upregulation on HeLa and $\mathrm{SiHa}$ cell viability was investigated using CCK-8 assay; miR-889 mimic transfection significantly inhibited HeLa and $\mathrm{SiHa}$ cell viability compared with cells transfected with miR-NC at 48 and $72 \mathrm{~h}$ post-transfection ( $\mathrm{P}<0.05$; Fig. 2B). The role of miR-889 transfection on cervical cancer cell invasion was also examined. HeLa and SiHa cells transfected with the miR-889 mimic exhibited a significantly reduced number of invading cells compared with miR-NC-transfected cells $(\mathrm{P}<0.05$; Fig. $2 \mathrm{C})$. These results suggested that miR-889 may serve a tumor suppressive role in cervical cancer.

FGFR2 is a direct target of miR-889 in cervical cancer cells. To decipher the mechanism of involvement in cervical cancer tumorigenesis, bioinformatic screens were used to predict the putative targets of miR-889. In particular,
FGFR2 was predicted to be a potential target of miR-889 according to TargetScan and miRDB (Fig. 3A). Since FGFR2 has been previously reported to be closely associated with cervical cancer tumorigenesis in addition to being directly targeted by a number of miRNAs in other malignancies $(23,26,28-30)$, it was chosen for further study. Luciferase reporter assay was used to determine if miR-889 can directly bind to the 3'-UTR of FGFR2 in cervical cancer cell lines. Compared with cells transfected with the miR-NC, miR-889 mimic overexpression significantly reduced luciferase activity in HeLa and SiHa cells transfected with the pMIR-FGFR2-3'-UTR WT plasmid $(\mathrm{P}<0.05)$, but exerted no significant effects on luciferase activity in cells transfected with the p-MIR-FGFR2-3'-UTR MUT plasmid (Fig. 3B). RT-qPCR and western blot analysis were subsequently performed to evaluate the effects of miR-889 mimic transfection on endogenous FGFR 2 expression in cervical cancer cells. The levels of FGFR2 mRNA (P<0.05; Fig. 3C) and protein $(\mathrm{P}<0.05$; Fig. 3D) expression were significantly reduced in HeLa and SiHa cells following miR-889 mimic transfection compared with cells transfected with miR-NC. These results suggested that FGFR2 is a direct target of miR-889 in cervical cancer cells.

FGFR2 knockdown mimics the suppressive effects of miR-889 overexpression on cervical cancer cell lines. Since FGFR2 was identified as a direct target of miR-889, the physiological function of FGFR2 was investigated in cervical cancer cell lines. FGFR2 siRNAs were chemically synthesized and transfected into HeLa and SiHa cells to suppress endogenous FGFR2 expression. Western blot analysis verified the efficiency of FGFR2 knockdown in HeLa and SiHa cells following FGFR2 siRNA transfection $(\mathrm{P}<0.05$; Fig. 4A). FGFR2 knockdown resulted in a significant reduction in HeLa and SiHa cell viability $(\mathrm{P}<0.05$; Fig. 4B) and invasive ability $(\mathrm{P}<0.05$; Fig. $4 \mathrm{C})$, an observation that was similar to that caused by miR-889 overexpression (Fig. 2). These results 


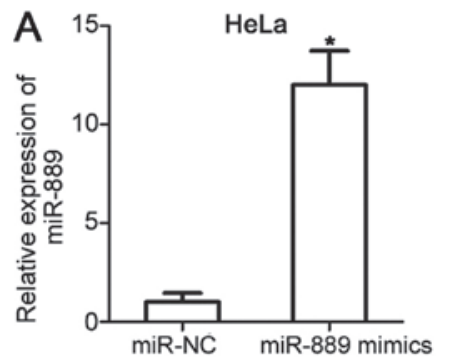

B
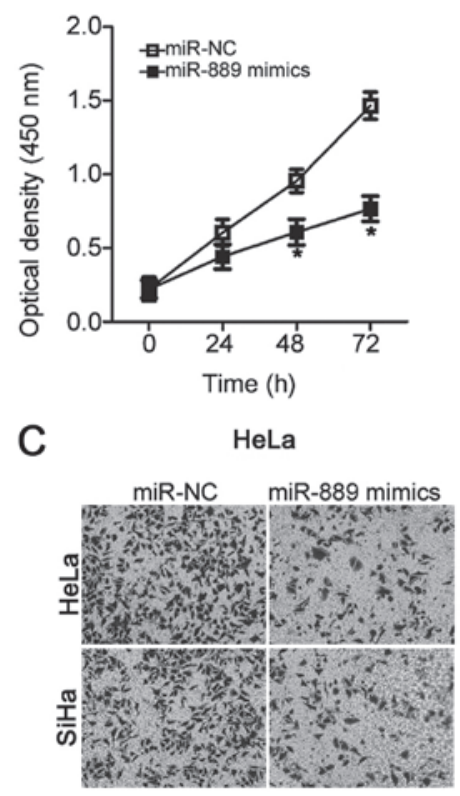
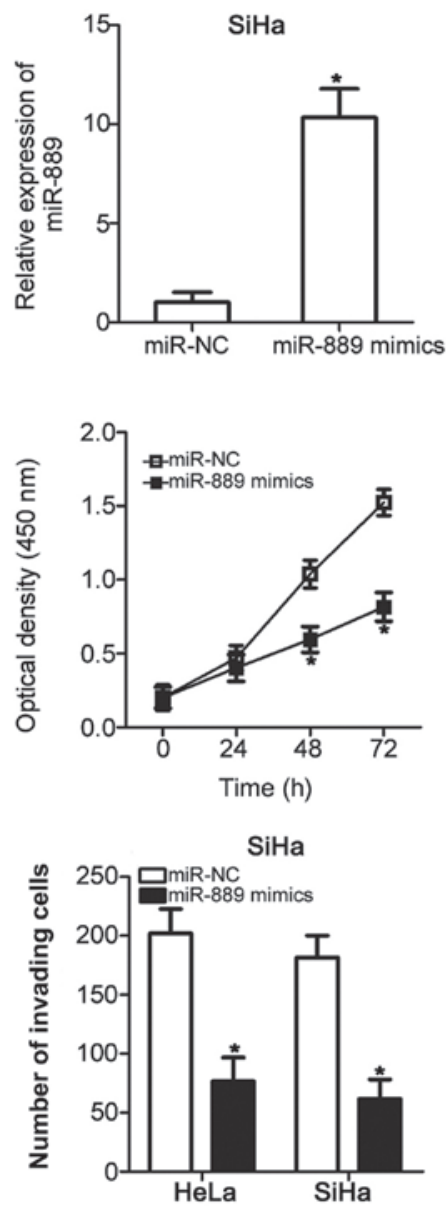

Figure 2. Transfection with miR-889 mimic exhibits inhibitory effects on HeLa and SiHa cell viability and invasion. (A) HeLa and SiHa cells were transfected with miR-889 mimic or miR-NC, and the increased expression of miR-889 was confirmed using reverse transcription-quantitative PCR. (B) Cell Counting Kit-8 assay was used to measure $\mathrm{HeLa}$ and $\mathrm{SiHa}$ cell viability following transfection with miR-889 mimic or miR-NC transfection. (C) Matrigel invasion assay was used to investigate the invasive ability of HeLa and SiHa cells transfected with miR- 889 mimic or miR-NC. ${ }^{*} \mathrm{P}<0.05$ vs. miR-NC. miR, microRNA; NC, negative control.

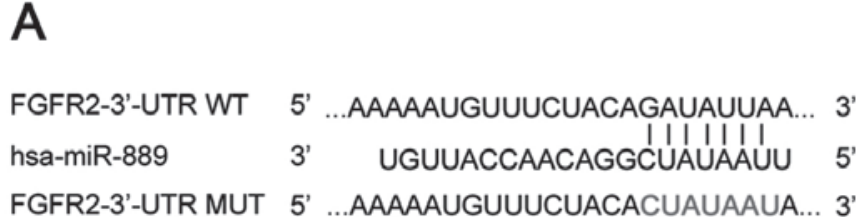

C

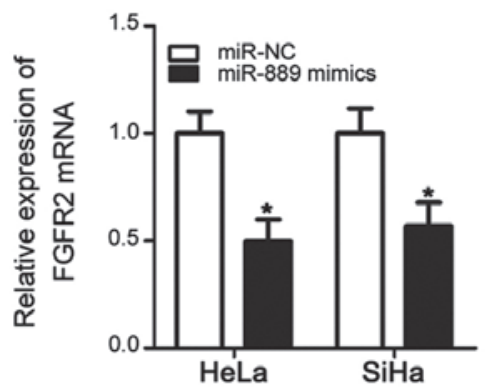

D
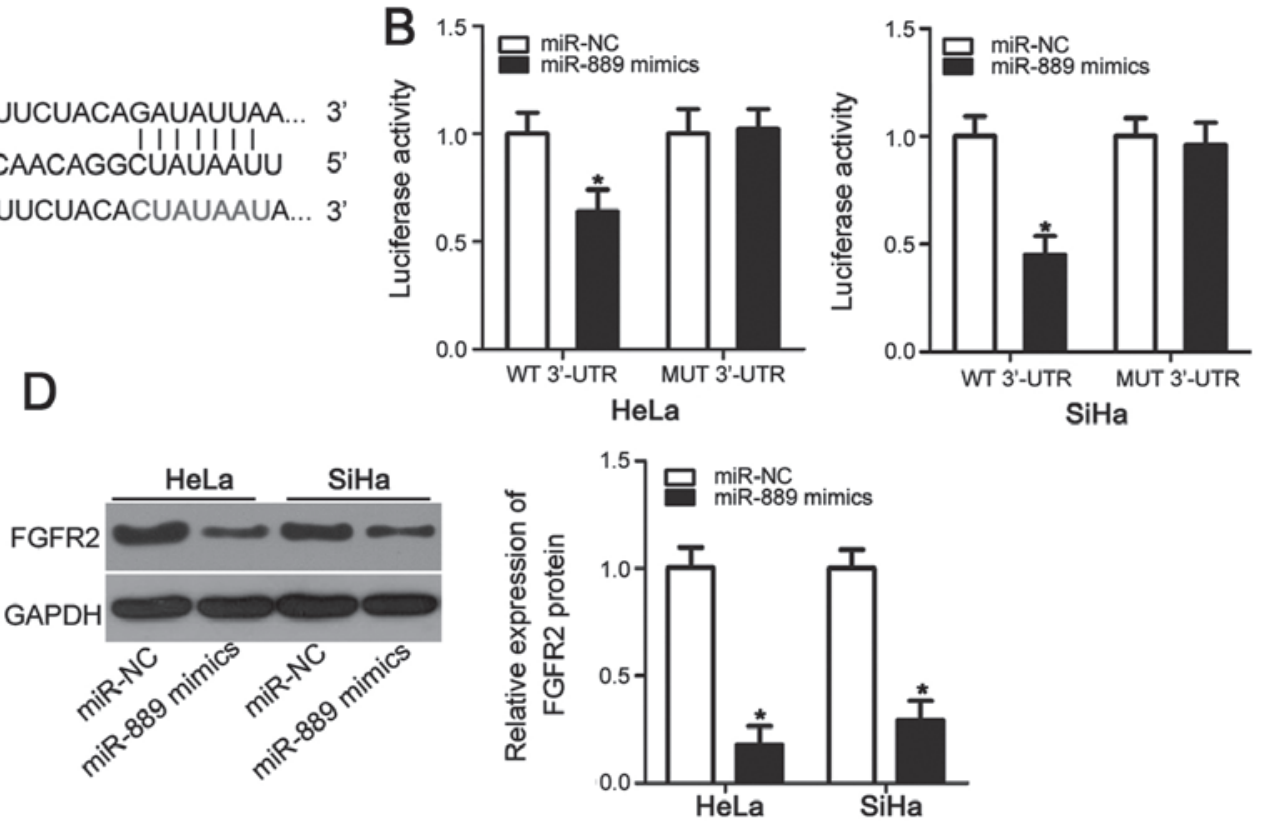

Figure 3. FGFR2 is the direct target of miR-889 in cervical cancer cells. (A) Sequence alignment between miR-889 and FGFR2-3'-UTR WT and FGFR2-3'-UTR MUT. (B) HeLa and SiHa cells were co-transfected with luciferase reporter plasmids encoding FGFR2-3'-UTR WT or FGFR2-3'-UTR MUT and miR-889 mimic or miR-NC. Luciferase reporter assay was carried out after $48 \mathrm{~h}$ transfection. (C) mRNA and (D) protein expression levels of FGFR2 were analyzed in $\mathrm{HeLa}$ and SiHa cells transfected with miR-889 mimic or miR-NC using reverse transcription-quantitative PCR and western blot analysis, respectively. ${ }^{*} \mathrm{P}<0.05$ vs. miR-NC. 3'-UTR, 3'untranslated region; FGFR2, fibroblast growth factor receptor 2; miR, microRNA; MUT, mutant; NC, negative control; WT, wild-type. 

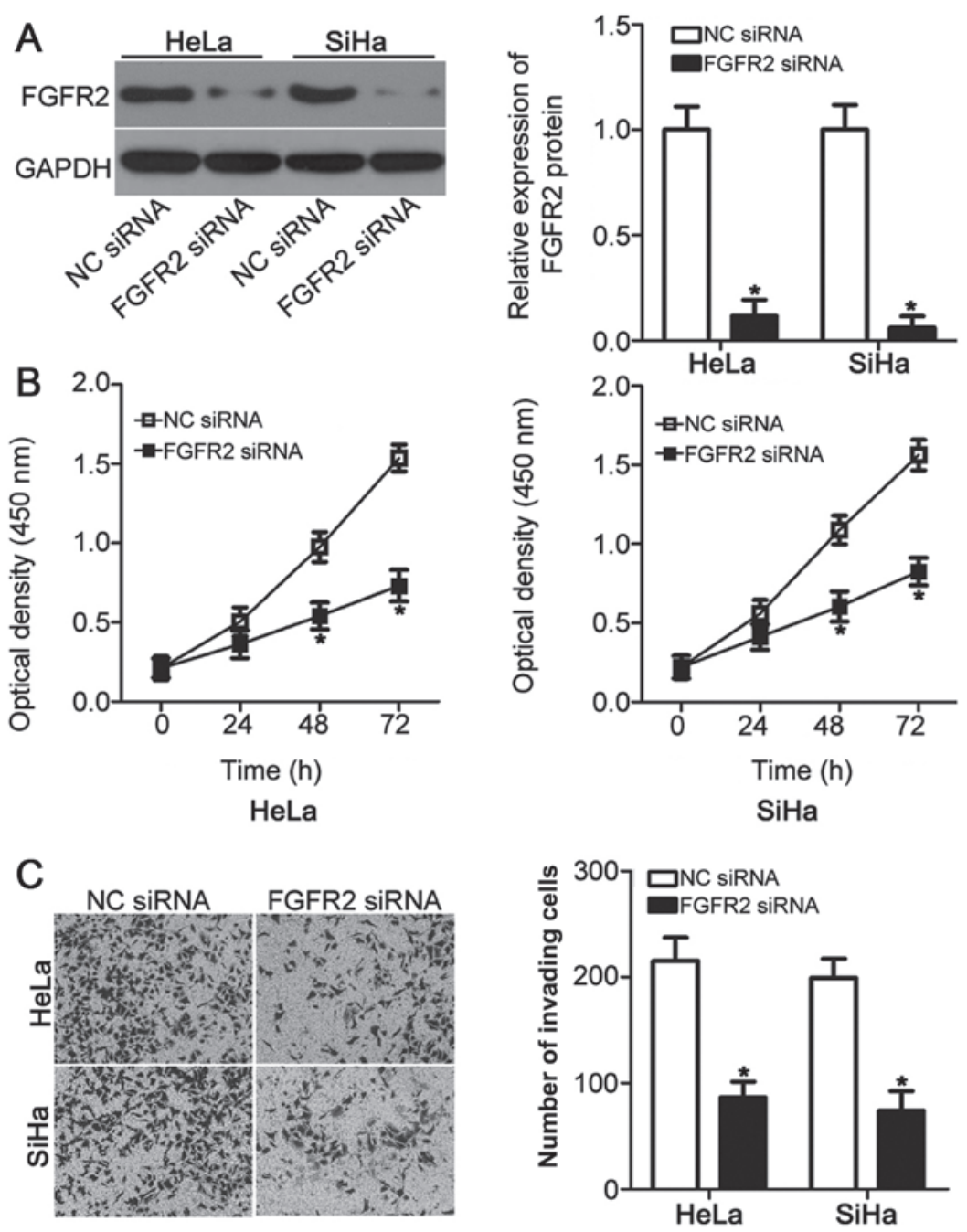

Figure 4. FGFR2 silencing inhibits HeLa and SiHa cell viability and invasion. (A) Western blot analysis for FGFR2 expression in HeLa and SiHa cells transfected with FGFR2 siRNA or NC siRNA. (B) HeLa and SiHa cell viability following transfection with FGFR2 siRNA or NC siRNA was evaluated using Cell Counting Kit-8 assay. (C) Matrigel invasion assay was used to measure HeLa and SiHa cell invasive ability following transfection with FGFR2 siRNA or NC siRNA. "P<0.05 vs. NC siRNA. miR, microRNA; NC, negative control; siRNA, small interfering RNA; FGFR2, fibroblast growth factor receptor 2.

further suggested that FGFR2 may be a functional target of miR-889 in cervical cancer cells.

FGFR2 overexpression rescues miR-889-mediated suppression of cervical cancer cell viability and invasion. To explore whether FGFR2 is a direct downstream mediator of miR-889, rescue experiments were performed in $\mathrm{HeLa}$ and $\mathrm{SiHa}$ cells by co-transfecting cells with pc-FGFR2 overexpression plasmids. First, western blot analysis confirmed that FGFR2 protein expression was significantly upregulated in $\mathrm{HeLa}$ and $\mathrm{SiHa}$ cells transfected with pc-FGFR2 compared with pcDNA3.1 empty vector $(\mathrm{P}<0.05$; Fig. 5A). Subsequently, HeLa and $\mathrm{SiHa}$ cells were co-transfected with miR-889 mimic and either pc-FGFR2 or empty pcDNA3.1 vector. Western blot analysis demonstrated that miR-889 mimic-induced suppression of FGFR2 expression was rescued in $\mathrm{HeLa}$ and $\mathrm{SiHa}$ cells co-transfected with pc-FGFR2, but not in those transfected with pcDNA3.1 ( $\mathrm{P}<0.05$; Fig. 5B). Similarly, FGFR2 overexpression reversed the suppressive effects of miR-889 mimic on $\mathrm{HeLa}$ and SiHa cell viability $(\mathrm{P}<0.05$; Fig. $5 \mathrm{C})$ and invasive ability ( $\mathrm{P}<0.05$; Fig. 5D). Taken together, these results further suggested that FGFR2 may be at least partially involved in the miR-889-mediated suppression of cervical cancer cell viability and invasion.

\section{Discussion}

miRNAs have regularly been found to be dysregulated in cervical cancer (31). Indeed, the dysregulation of miRNAs has been reported to contribute to a number of processes associated with cancer pathophysiology, including cell proliferation, cell cycle, apoptosis, epithelial-mesenchymal transition, metastasis, angiogenesis and resistance to chemoand radiotherapy (32-34). Therefore, a thorough understanding of the mechanism underlying cervical cancer carcinogenesis and progression is crucial for early diagnosis and improving patient outcome (14). In particular, further exploration on the specific roles of dysregulated miRNAs in cervical cancer and their associated mechanism may provide an avenue for the development of effective therapeutic approaches.

miR-889 is upregulated in esophageal squamous cell carcinoma tissues and cell lines (20). Upregulation of miR-889 
A

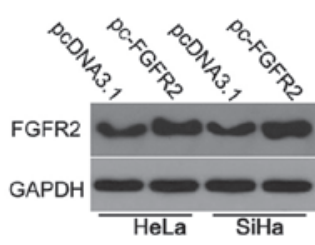

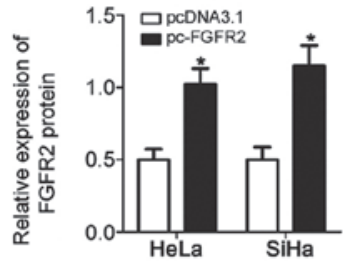

C $\quad$ - miR-NC

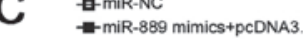

- - miR-889 mimics+pc-FGFR2
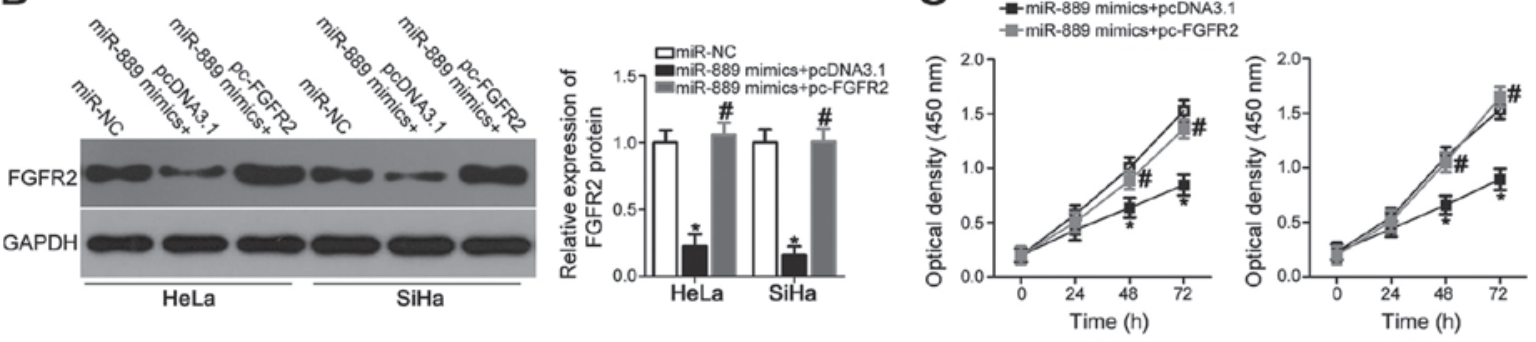

D
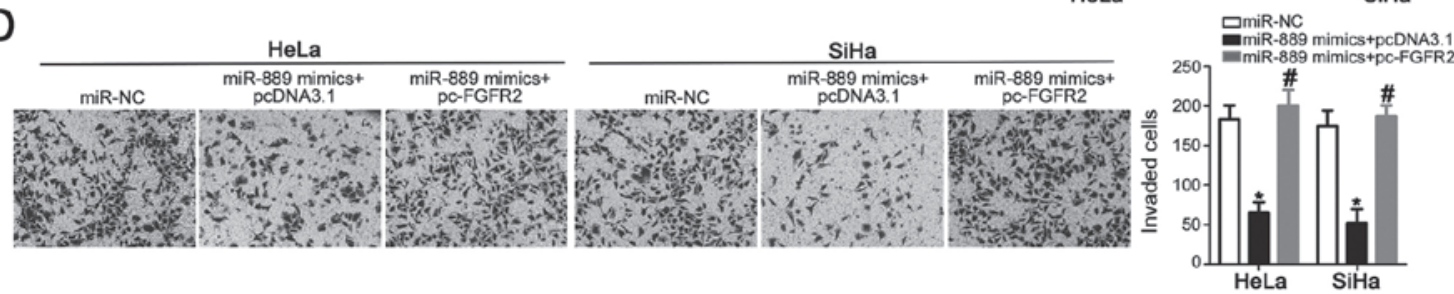

Figure 5. FGFR2 overexpression rescues miR-889-mediated suppression of cervical cancer cell viability and invasion. (A) pc-FGFR2 or empty pcDNA3.1 plasmid was transfected into HeLa and SiHa cells, and FGFR2 protein expression was determined using western blot analysis. "P<0.05 vs. pcDNA3.1. (B) pc-FGFR2 or pcDNA3.1 was co-transfected with miR-889 mimic in HeLa and SiHa cells. Western blot analysis was used to measure FGFR2 protein expression $72 \mathrm{~h}$ following transfection. ${ }^{*} \mathrm{P}<0.05$ vs. miR-NC; ${ }^{*} \mathrm{P}<0.05$ vs. miR- 889 mimic + pcDNA3.1. (C) Cell viability and (D) invasive ability of HeLa and $\mathrm{SiHa}$ cells were assessed using Cell Counting Kit-8 and Matrigel invasion assays, respectively. ${ }^{\text {"P }}<0.05$ vs. miR-NC; ${ }^{~} \mathrm{P}<0.05$ vs. miR-889 mimic + pcDNA3.1. FGFR2, fibroblast growth factor receptor 2; miR, microRNA; NC, negative control; pc-FGFR2, pcDNA3.1-FGFR2; siRNA, small interfering RNA.

expression prohibits esophageal squamous cell carcinoma cell growth in vitro and in vivo by directly targeting disabled homolog-2 interactive protein (20). miR-889 is also downregulated by histone deacetylase inhibitors in hepatocellular carcinoma cells (21). miR-889 overexpression attenuates hepatocellular carcinoma cell susceptibility to natural killer cell-mediated lysis by downregulating major histocompatibility complex class I chain-related gene B (21). However, the role of miR-889 in cervical cancer remains unclear. The present study revealed that miR-889 expression was lower in cervical cancer tissues and was found to be negatively associated with FIGO stages and lymph node metastasis. miR-889 overexpression inhibited viability and invasive ability of cervical cancer cell lines. These results suggested that miR-889 may be an attractive therapeutic target for patients with this malignancy.

miRNAs exert their functions by directly targeting different genes to regulate cellular processes (11). Specifically, identifying the direct targets of miR-889 in cervical cancer is important for elucidating their action on the malignant signatures, which may be useful for the development of novel therapeutic interventions. Therefore, to explore the mechanism in which miR-889 attenuated the malignant phenotype of cervical cancer cells, a bioinformatics screen was performed to search for genes directly targeted by miR-889. This screen identified FGFR2 as a potential miR-889 target, which was subsequently validated using luciferase reporter assay. Expression analysis of mRNA and protein levels found that
miR-889 upregulation markedly suppressed FGFR2 expression in cervical cancer cell lines. Supporting this, the effects of miR-889 overexpression on the same cell lines could be successfully mimicked by FGFR2 knockdown. Finally, FGFR2 overexpression abrogated the suppressive effects of miR-889 on cervical cancer cell viability and invasion. These findings strongly suggested that FGFR2 is a direct and functional downstream target of miR-889 in cervical cancer cells.

The FGFR2 gene, located on human chromosome 10q26, is a member of the FGF2/FGFR2 signaling pathway (35). Previous studies have reported that FGFR2 is highly expressed in a number of human cancers, including gastric (22), thyroid (23), bladder (24) and breast cancer (25). FGFR2 serves a role in tumorigenesis by regulating a number of physiological processes, including cell growth, apoptosis, survival, metastasis, motility and angiogenesis (36). FGFR2 is expressed strongly in cervical cancer tissues and cell lines in addition to associating with lymph node metastasis in previous studies $(26,28)$. Results from the present study indicated that miR-889 directly targeted FGFR2 to suppress the malignant phenotype of cervical cancer. Based on these data, the miR-889/FGFR2 axis may serve as a potential therapeutic target for patients with cervical cancer in the future.

In conclusion, to the best of our knowledge, this was the first study to demonstrate the reduction of miR-889 expression in cervical cancer tissues and cell lines, which was associated with FIGO stages and lymph node metastasis. miR-889 overexpression suppressed cervical cancer cell viability and invasion 
by directly targeting FGFR2. Therefore, miR-889 may serve important roles in cervical cancer physiology, suggesting this miRNA to be a potentially novel therapeutic target. However, the correlation between miR-889 and the prognosis of patients with cervical cancer was not analyzed in this study; which is a limitation of this study. In future investigations, prognosis data of patients with cervical cancer will be collected to explore the association between miR-889 expression and prognosis.

\section{Acknowledgements}

Not applicable.

\section{Funding}

No funding was received.

\section{Availability of data and materials}

The datasets used and/or analyzed during the present study are available from the corresponding author upon reasonable request.

\section{Authors' contributions}

$\mathrm{KH}$ designed this research and performed reverse transcription-quantitative PCR and statistical analysis. YS and YC conducted Cell Counting Kit-8 and invasion assays. Dual-luciferase reporter assays and western blot analyses were carried out by YZ. All authors read and approved the final draft of the manuscript.

\section{Ethics approval and consent to participate}

The present study was approved by the Ethics Committee of Weifang People's Hospital (Weifang, China), and was performed in accordance with the Declaration of Helsinki and guidelines of the Ethics Committee of Weifang People's Hospital. Written informed consent was provided by all individuals that participated in this study.

\section{Patient consent for publication}

Not applicable.

\section{Competing interests}

The authors declare that they have no competing interests.

\section{References}

1. Siegel RL, Miller KD and Jemal A: Cancer Statistics, 2017. CA Cancer J Clin 67: 7-30, 2017.

2. Bray F, Ferlay J, Soerjomataram I, Siegel RL, Torre LA and Jemal A: Global cancer statistics 2018: GLOBOCAN estimates of incidence and mortality worldwide for 36 cancers in 185 countries. CA Cancer J Clin 68: 394-424, 2018.

3. Castellsague X: Natural history and epidemiology of HPV infection and cervical cancer. Gynecol Oncol 110 (3 Suppl 2): S4-S7, 2008.

4. Du CX and Wang Y: Expression of P-Akt, NFkappaB and their correlation with human papillomavirus infection in cervical carcinoma. Eur J Gynaecol Oncol 33: 274-277, 2012.
5. Ghebre RG, Grover S, Xu MJ, Chuang LT and Simonds H: Cervical cancer control in HIV-infected women: Past, present and future. Gynecol Oncol Rep 21: 101-108, 2017.

6. Hildesheim A and Wang SS: Host and viral genetics and risk of cervical cancer: A review. Virus Res 89: 229-240, 2002.

7. Link A and Kupcinskas J: MicroRNAs as non-invasive diagnostic biomarkers for gastric cancer: Current insights and future perspectives. World J Gastroenterol 24: 3313-3329, 2018.

8. To KK, Tong CW, Wu M and Cho WC: MicroRNAs in the prognosis and therapy of colorectal cancer: From bench to bedside. World J Gastroenterol 24: 2949-2973, 2018.

9. Wang F, Li B and Xie X: The roles and clinical significance of microRNAs in cervical cancer. Histol Histopathol 31: 131-139, 2016.

10. Bartel DP: MicroRNAs: Target recognition and regulatory functions. Cell 136: 215-233, 2009.

11. He L and Hannon GJ: MicroRNAs: small RNAs with a big role in gene regulation. Nat Rev Genet 5: 522-531, 2004.

12. Kozomara A and Griffiths-Jones S: miRBase: Annotating high confidence microRNAs using deep sequencing data. Nucleic Acids Res 42: D68-D73, 2014.

13. Friedman RC, Farh KK, Burge CB and Bartel DP: Most mammalian mRNAs are conserved targets of microRNAs. Genome Res 19: 92-105, 2009.

14. Laengsri V, Kerdpin U, Plabplueng C, Treeratanapiboon L and Nuchnoi P: Cervical Cancer Markers: Epigenetics and microRNAs. Lab Med 49: 97-111, 2018.

15. Zhu B, Ju S, Chu H, Shen X, Zhang Y, Luo X and Cong H: The potential function of microRNAs as biomarkers and therapeutic targets in multiple myeloma. Oncol Lett 15: 6094-6106, 2018

16. Lu J, Zhan Y, Feng J, Luo J and Fan S: MicroRNAs associated with therapy of non-small cell lung cancer. Int J Biol Sci 14: 390-397, 2018.

17. Cai N, Hu L, Xie Y, Gao JH, Zhai W, Wang L, Jin QJ, Qin CY and Qiang R: MiR-17-5p promotes cervical cancer cell proliferation and metastasis by targeting transforming growth factor-beta receptor 2. Eur Rev Med Pharmacol Sci 22: 1899-1906, 2018

18. Li GC, Cao XY, Li YN, Qiu YY, Li YN, Liu XJ and Sun XX: MicroRNA-374b inhibits cervical cancer cell proliferation and induces apoptosis through the p38/ERK signaling pathway by binding to JAM-2. J Cell Physiol 233: 7379-7390, 2018.

19. Sanches JGP, Xu Y, Yabasin IB, Li M, Lu Y, Xiu X, Wang L, Mao L, Shen J, Wang B, et al: miR-501 is upregulated in cervical cancer and promotes cell proliferation, migration and invasion by targeting CYLD. Chem Biol Interact 285: 85-95, 2018.

20. Xu Y, He J, Wang Y, Zhu X, Pan Q, Xie Q and Sun F: miR-889 promotes proliferation of esophageal squamous cell carcinomas through DAB2IP. FEBS Lett 589: 1127-1135, 2015.

21. Xie H, Zhang Q, Zhou H, Zhou J, Zhang J, Jiang Y, Wang J, Meng X, Zeng L and Jiang X: microRNA-889 is downregulated by histone deacetylase inhibitors and confers resistance to natural killer cytotoxicity in hepatocellular carcinoma cells. Cytotechnology 70: 513-521, 2018.

22. Cha Y, Kim HP, Lim Y, Han SW, Song SH and Kim TY: FGFR2 amplification is predictive of sensitivity to regorafenib in gastric and colorectal cancers in vitro. Mol Oncol 12: 993-1003, 2018.

23. Fu YT,Zheng HB,Zhang DQ,Zhou L and Sun H: MicroRNA-1266 suppresses papillary thyroid carcinoma cell metastasis and growth via targeting FGFR2. Eur Rev Med Pharmacol Sci 22: 3430-3438, 2018

24. Marzioni D, Lorenzi T, Mazzucchelli R, Capparuccia L, Morroni M, Fiorini R, Bracalenti C, Catalano A, David G, Castellucci M, et al: Expression of basic fibroblast growth factor, its receptors and syndecans in bladder cancer. Int J Immunopathol Pharmacol 22: 627-638, 2009.

25. Lei H and Deng CX: Fibroblast growth factor receptor 2 signaling in breast cancer. Int J Biol Sci 13: 1163-1171, 2017.

26. Choi CH, Chung JY, Kim JH, Kim BG and Hewitt SM: Expression of fibroblast growth factor receptor family members is associated with prognosis in early stage cervical cancer patients. J Transl Med 14: 124, 2016.

27. Livak KJ and Schmittgen TD: Analysis of relative gene expression data using real-time quantitative PCR and the 2(-Delta Delta C(T)) method. Methods 25: 402-408, 2001

28. Kawase R, Ishiwata T, Matsuda Y, Onda M, Kudo M, Takeshita T and Naito Z: Expression of fibroblast growth factor receptor 2 IIIc in human uterine cervical intraepithelial neoplasia and cervical cancer. Int J Oncol 36: 331-340, 2010.

29. Li M, Qian Z, Ma X, Lin X, You Y, Li Y, Chen T and Jiang H: MiR-628-5p decreases the tumorigenicity of epithelial ovarian cancer cells by targeting at FGFR2. Biochem Biophys Res Commun 495: 2085-2091, 2018. 
30. Yang X, Ruan H,Hu X, Cao A and Song L: miR-381-3p suppresses the proliferation of oral squamous cell carcinoma cells by directly targeting FGFR2. Am J Cancer Res 7: 913-922, 2017.

31. Wang JY and Chen LJ: The role of miRNAs in the invasion and metastasis of cervical cancer. Biosci Rep 39: 2019

32. Pedroza-Torres A, Lopez-Urrutia E, Garcia-Castillo V, Jacobo-Herrera N, Herrera LA, Peralta-Zaragoza O, López-Camarillo C, De Leon DC, Fernández-Retana J, Cerna-Cortés JF and Pérez-Plasencia C: MicroRNAs in cervical cancer: Evidences for a miRNA profile deregulated by HPV and its impact on radio-resistance. Molecules 19: 6263-6281, 2014.

33. Banno K, Iida M, Yanokura M, Kisu I, Iwata T, Tominaga E, Tanaka K and Aoki D: MicroRNA in cervical cancer: OncomiRs and tumor suppressor miRs in diagnosis and treatment. TheScientificWorldJournal 2014: 178075, 2014.
34. Shishodia G, Verma G, Das BC and Bharti AC: miRNA as viral transcription tuners in HPV-mediated cervical carcinogenesis. Front Biosci (Schol Ed) 10: 21-47, 2018

35. Katoh Y and Katoh M: FGFR2-related pathogenesis and FGFR2-targeted therapeutics (Review). Int J Mol Med 23: 626-639, 2018

36. Ishiwata T: Role of fibroblast growth factor receptor-2 splicing in normal and cancer cells. Front Biosci (Landmark Ed) 23 626-639, 2018. 